\title{
A MODIFIED AOR-TYPE ITERATIVE METHOD FOR $L$-MATRIX LINEAR SYSTEMS
}

\author{
SHI-LIANG WU ${ }^{1}$ and TING-ZHU HUANG ${ }^{\oplus 1}$
}

(Received 14 April, 2007; revised 22 October, 2007)

\begin{abstract}
Both Evans et al. and Li et al. have presented preconditioned methods for linear systems to improve the convergence rates of AOR-type iterative schemes. In this paper, we present a new preconditioner. Some comparison theorems on preconditioned iterative methods for solving $L$-matrix linear systems are presented. Comparison results and a numerical example show that convergence of the preconditioned Gauss-Seidel method is faster than that of the preconditioned AOR iterative method.
\end{abstract}

2000 Mathematics subject classification: primary 65F10; secondary $15 \mathrm{~A} 06$.

Keywords and phrases: preconditioner; $L$-matrix; AOR method; spectral radius.

\section{Introduction}

The solutions of many problems in mathematics, physics, fluid dynamics, engineering and so on are eventually turned into the solutions of large linear systems of the form

$$
A x=b,
$$

where $A \in R^{n \times n}$ and $b \in R^{n}$ are given and $x \in R^{n}$ is unknown. For example, it is shown in [8] that using a finite-difference scheme for the discretization of the Poisson equation leads to linear system (1.1).

For any splitting $A=M-N$ with $\operatorname{det}(M) \neq 0$, the basic iterative method for solving (1.1) is

$$
x^{(n+1)}=M^{-1} N x^{(n)}+M^{-1} b, \quad n=0,1, \cdots
$$

\footnotetext{
'School of Applied Mathematics, University of Electronic Science and Technology of China, Chengdu, Sichuan,610054, P. R. China; email: wushiliang1999@126.com, tzhuang@uestc.edu.cn, tingzhuhuang@126.com.

(C) Australian Mathematical Society 2007, Serial-fee code 1446-1811/07
} 
under the assumption that $a_{i i} \neq 0, i=1,2, \ldots, n$. For simplicity, without loss of generality, we assume throughout this paper that

$$
A=I-L-U \text {, }
$$

where $I$ is the identity matrix, and $L$ and $U$ are strictly lower and upper triangular matrices obtained from $A$, respectively. Then the iteration matrices of the classical AOR iterative method in [4] are defined by

$$
L_{r w}=(I-r L)^{-1}[(1-w) I+(w-r) L+w U]
$$

where $w$ and $r$ are real parameters with $w \neq 0$. It is easy to get the Jacobi, the GaussSeidel (GS) and the successive overrelaxation (SOR) methods for certain values of $w$ and $r$.

In order to accelerate the convergence of any iterative method solving the linear system (1.1), some techniques of preconditioning have been used. That is,

$$
P A x=P b,
$$

where $P$, called the preconditioner, is a non-singular matrix. The preconditioned system (1.3) with different preconditioners $P$ is considered in [2], [4-7] and [9].

Recently, in [2], Evans et al. presented a preconditioned AOR iterative method by using the preconditioners $P=I+S$ and $P=I+S^{\prime}$, where

$$
S=\left[\begin{array}{cccc}
0 & 0 & \cdots & 0 \\
0 & 0 & \cdots & 0 \\
\vdots & \vdots & \ddots & \vdots \\
-a_{n 1} & 0 & \ldots & 0
\end{array}\right] \text { and } S^{\prime}=\left[\begin{array}{cccc}
0 & 0 & \ldots & -a_{1 n} \\
0 & 0 & \ldots & 0 \\
\vdots & \vdots & \ddots & \vdots \\
0 & 0 & \ldots & 0
\end{array}\right]
$$

The authors suggest that if the original iteration matrix is non-negative irreducible, then performing Gaussian elimination on the last element of the first column of the iteration matrix (or the last element of the first row of the iteration matrix) to make it zero will improve the convergence of the iteration matrix and showed that the preconditioned AOR method is better than the original one.

In [6], Li et al. considered as a preconditioner $P=I+\bar{S}$, where

$$
\vec{S}=\left[\begin{array}{ccccc}
0 & -a_{12} & 0 & \cdots & 0 \\
0 & 0 & -a_{23} & \cdots & 0 \\
\vdots & \vdots & \vdots & \cdots & \vdots \\
0 & 0 & 0 & \cdots & -a_{n-1 n} \\
0 & 0 & 0 & \cdots & 0
\end{array}\right],
$$


whose effect on $A$ is to eliminate the elements of the first upper diagonal in order to improve the convergence rate of the AOR iteration method.

In this paper, a new preconditioner different from those considered by Evans $e t$ al. and $\mathrm{Li}$ et al. for AOR-type iterative methods for solving consistent linear systems is presented, which is the preconditioner $P=I+S+\bar{S}$. Some comparison theorems on preconditioned iterative methods for solving $L$-matrix linear systems are presented. Comparison results show that convergence of the preconditioned Gauss-Seidel method is faster than that of the preconditioned AOR (or SOR) iterative method. The numerical examples also show that convergence of our preconditioned AOR scheme is faster than that in [2] and [6].

\section{Preliminaries}

For convenience, the following definitions and lemmas are useful in this paper. Here $\rho(\cdot)$ denotes the spectral radius of a matrix.

DEFINITION 2.1. [10] Matrix $A$ is an L-matrix if $a_{i i}>0 ; i=1, \cdots, n$ and $a_{i j} \leq 0$, for all $i, j=1,2, \cdots, n ; i \neq j$.

DEFINITION 2.2. [8] Matrix $A$ is irreducible if the directed graph associated to $A$ is strongly connected.

DEFINITION 2.3. [8] The representation $A=M-N$ is called a regular splitting of $A$ if $M^{-1} \geq 0$ and $N \geq 0$.

LeMMA 2.4. [8] Let $A \in C^{n \times n}$ be a nonnegative and irreducible $n \times n$ matrix. Then

(i) A has a positive real eigenvalue equal to its spectral radius $\rho(A)$;

(ii) There exists an eigenvector $x>0$ corresponding to $\rho(A)$;

(iii) $\rho(A)$ is a simple eigenvalue of $A$;

(iv) $\rho(A)$ increases when any entry of $A$ increases.

LEMMA 2.5. [1] Let A be a nonnegative matrix. Then

(1) If $\alpha x \leq A x$ for some nonnegative vector $x, x \neq 0$, then $\alpha \leq \rho(A)$.

(2) If $A x \leq \beta x$ for some positive vector $x$, then $\rho(A) \leq \beta$. Moreover, if $A$ is irreducible and if $0 \neq \alpha x \leq A x \leq \beta x$ for some nonnegative vector $x$, then

$$
\alpha \leq \rho(A) \leq \beta
$$

and $x$ is a positive vector. 
LEMMA 2.6. [8] Let $A=M_{1}-N_{1}=M_{2}-N_{2}$ be two regular splittings of $A$, where $A^{-1} \geq 0$. If $N_{2} \geq N_{1} \geq 0$, then

$$
0 \leq \rho\left(M_{1}^{-1} N_{1}\right) \leq \rho\left(M_{2}^{-1} N_{2}\right)<1 .
$$

If, moreover, $A^{-1}>0$ and if $N_{2} \geq N_{1} \geq 0$, equality excluded, then

$$
0<\rho\left(M_{1}^{-1} N_{1}\right)<\rho\left(M_{2}^{-1} N_{2}\right)<1 .
$$

\section{A preconditioned AOR iterative method}

Now, let us consider the preconditioned linear system,

$$
\widetilde{A} x=\widetilde{b},
$$

where $\tilde{A}=(I+\tilde{S}) A$ and $\widetilde{b}=(I+\tilde{S}) b$ with

$$
\tilde{S}=\left[\begin{array}{ccccc}
0 & -a_{12} & 0 & \cdots & 0 \\
0 & 0 & -a_{23} & \cdots & 0 \\
\vdots & \vdots & \vdots & \cdots & \vdots \\
0 & 0 & 0 & \cdots & -a_{n-1, n} \\
-a_{n 1} & 0 & 0 & \cdots & 0
\end{array}\right]
$$

We express the coefficient matrix of (3.1) as

$$
\widetilde{A}=\widetilde{D}-\widetilde{L}-\widetilde{U}
$$

where $\widetilde{D}=\operatorname{diag}(\tilde{A})$ and $\tilde{L}$ and $\widetilde{U}$ are strictly lower and upper triangular matrices obtained from $\widetilde{A}$, respectively. By calculation, we obtain that

$$
\begin{aligned}
& \tilde{D}=\left[\begin{array}{cccc}
1-a_{12} a_{21} & 0 & \cdots & 0 \\
0 & 1-a_{23} a_{32} & \cdots & 0 \\
\vdots & \vdots & \ddots & \vdots \\
0 & 0 & \cdots & 1-a_{1 n} a_{n 1}
\end{array}\right] \\
& \tilde{L}=\left[\begin{array}{ccccc}
0 & 0 & 0 & \cdots & \cdots \\
-a_{21}+a_{23} a_{31} & 0 & 0 & \cdots & 0 \\
\vdots & \vdots & \ddots & \vdots & \vdots \\
-a_{n-1,1}+a_{n-1, n} a_{n 1} & -a_{n-1,2}+a_{n-1, n} a_{n 2} & \cdots & 0 & 0 \\
0 & -a_{n 2}+a_{n 1} a_{12} & \cdots & -a_{n, n-1}+a_{n 1} a_{1, n-1} & 0
\end{array}\right]
\end{aligned}
$$


and

$$
\widetilde{U}=\left[\begin{array}{ccccc}
0 & 0 & -a_{13}+a_{12} a_{23} & \ldots & -a_{1 n}+a_{12} a_{2 n} \\
0 & 0 & 0 & \cdots & -a_{2 n}+a_{23} a_{3 n} \\
\vdots & \vdots & \ddots & \ddots & \vdots \\
0 & 0 & 0 & \ddots & 0 \\
0 & 0 & 0 & \cdots & 0
\end{array}\right]
$$

Applying the AOR method to the preconditioned linear system (3.1), we have the corresponding preconditioned AOR iterative method whose iterative matrix is

$$
\widetilde{L}_{r u}=(\widetilde{D}-r \widetilde{L})^{-1}[(1-w) \widetilde{D}+(w-r) \widetilde{L}+w \widetilde{U}]
$$

REMARK 1. When $a_{n 1}=0, \tilde{S}$ is considered in [6]. When $a_{i, i+1}=0, i=$ $1,2, \ldots, n-1, \widetilde{S}$ is considered in [2].

\section{Main results}

First, we need the following lemmas for our proof.

LEMMA 4.1. Let $A$ and $\tilde{A}$ be the coefficient matrices of the linear systems (1.1) and (3.1), respectively. If $0 \leq r \leq w \leq 1(w \neq 0, r \neq 1)$, A is an irreducible L-matrix with $0<a_{1 n} a_{n 1}<1$ and there exists a nonempty set of $\alpha \subseteq N=\{1,2, \cdots, n-1\}$ such that

$$
\begin{cases}0<a_{i, i+1} a_{i+1, i}<1, & i \in \alpha, \\ a_{i, i+1} a_{i+1, i}=0, & i \in N \backslash \alpha .\end{cases}
$$

Then the iterative matrices $L_{r w}$ and $\tilde{L}_{r w}$ associated to the AOR method applied to the linear systems (1.1) and (3.1), respectively, are nonnegative and irreducible.

PROOF. Since $A$ is an $L$-matrix, we have that $L \geq 0(U \geq 0)$ is a strictly lower (upper) triangular matrix. So $(I-r L)^{-1}=I+r L+r^{2} L^{2}+\cdots+r^{n-1} L^{n-1} \geq 0$.

By (1.2), we have

$$
\begin{aligned}
L_{r w}= & (I-r L)^{-1}[(1-w) I+(w-r) L+w U] \\
= & {\left[I+r L+r^{2} L^{2}+\cdots+r^{n-1} L^{n-1}\right][(1-w) I+(w-r) L+w U] } \\
= & (1-w) I+(w-r) L+w U+r L(1-w) I+r L[(w-r) L+w U] \\
& +\left(r^{2} L^{2}+\cdots+r^{n-1} L^{n-1}\right)[(1-w) I+(w-r) L+w U] \\
= & (1-w) I+w(1-r) L+w U+T,
\end{aligned}
$$


where

$$
\begin{aligned}
T= & r L[(w-r) L+w U] \\
& +\left(r^{2} L^{2}+\cdots+r^{n-1} L^{n-1}\right) \times[(1-w) I+(w-r) L+w U] \geq 0 .
\end{aligned}
$$

So $L_{r w}$ is nonnegative. We can also get that $(1-w) I+w(1-r) L+w U$ is irreducible since $A$ is irreducible, hence $L_{r w}$ is irreducible.

Subsequently, we prove that $\widetilde{L}_{w, r}$ is nonnegative and irreducible.

It is easy to get that $\widetilde{D} \geq 0$ from the conditions of Lemma 4.1. Since $A$ is an $L$-matrix, we get that $\widetilde{L} \geq 0$ and $\widetilde{U} \geq 0$. By (3.2), we have

$$
\begin{aligned}
\widetilde{L}_{r w} & =(\widetilde{D}-r \widetilde{L})^{-1}[(1-w) \widetilde{D}+(w-r) \tilde{L}+w \widetilde{U}] \\
& =\left(I-r \widetilde{D}^{-1} \widetilde{L}\right)^{-1}\left[(1-w) I+(w-r) \widetilde{D}^{-1} \widetilde{L}+w \widetilde{D}^{-1} \widetilde{U}\right] \\
& =(1-w) I+w(1-r) \widetilde{D}^{-1} \widetilde{L}+w \widetilde{D}^{-1} \tilde{U}+\widetilde{T}
\end{aligned}
$$

where

$$
\begin{aligned}
\widetilde{T}= & r \widetilde{D}^{-1} \widetilde{L}\left[(w-r) \widetilde{D}^{-1} \widetilde{L}+w \widetilde{D}^{-1} \widetilde{U}\right]+\left[r^{2}\left(\widetilde{D}^{-1} \widetilde{L}\right)^{2}+\cdots+r^{n-1}\left(\widetilde{D}^{-1} \widetilde{L}\right)^{n-1}\right] \\
& \times\left[(1-w) l+(w-r) \widetilde{D}^{-1} \widetilde{L}+w \tilde{D}^{-1} \tilde{U}\right] \geq 0 .
\end{aligned}
$$

So we have $\tilde{T} \geq 0$ and $\tilde{L}_{r w} \geq 0$ from $\widetilde{D} \geq 0, \tilde{L} \geq 0$ and $\tilde{U} \geq 0$. As $\tilde{L}_{r w}$, we have that $\widetilde{L}_{r w}$ is nonnegative and irreducible too.

We need the following equalities to prove Theorem 4.2 , which are easily proved:

(E1) $\tilde{U}=\bar{U}=\bar{S} U-\bar{S}+U$;

(E2) $\bar{D}-\bar{L}=I-L-\bar{S} L$;

(E3) $\tilde{D}-\tilde{L}=\bar{D}-\bar{L}+S-S U$.

THEOREM 4.2. Let $L_{r w}$ and $\tilde{L}_{r w}$ be the iterative matrices of the AOR method given by (1.2) and (3.2), respectively. If $0 \leq r \leq w \leq 1(w \neq 0, r \neq 1), A$ is an irreducible L-matrix with $0<a_{1 n} a_{n 1}<1$ and there exists a nonempty set of $\alpha \subseteq N=\{1,2, \ldots, n-1\}$ such that

$$
\begin{cases}0<a_{i, i+1} a_{i+1, i}<1, & i \in \alpha, \\ a_{i, i+1} a_{i+1, i}=0, & i \in N \backslash \alpha .\end{cases}
$$

Then

(1) $\rho\left(\tilde{L}_{r w}\right)<\rho\left(L_{r w}\right)$ if $\rho\left(L_{r w}\right)<1$;

(2) $\rho\left(\tilde{L}_{r w}\right)=\rho\left(L_{r w}\right)$, if $\rho\left(L_{r w}\right)=1$;

(3) $\rho\left(\tilde{L}_{r w}\right)>\rho\left(L_{r w}\right)$, if $\rho\left(L_{r w}\right)>1$. 
Proof. From Lemma 4.1 , it is clear that $L_{r w}$ and $\tilde{L}_{r w}$ are nonnegative and irreducible matrices. Thus, from Lemma 2.4 there exists a positive:vector $x$, such that

$$
L_{r w} x=\lambda x
$$

where $\lambda=\rho\left(L_{r w}\right)$ or, equivalently,

$$
[(1-w) l+(w-r) L+w U] x=\lambda(I-r L) x .
$$

From (4.1), we have

$$
w U x=(\lambda+w-1) x+(r-w-\lambda r) L x .
$$

Therefore, for this $x>0$,

$$
\tilde{L}_{r w} x-\lambda x=(\tilde{D}-r \tilde{L})^{-1}[(1-w) \tilde{D}+(w-r) \tilde{L}+w \tilde{U}-\lambda(\tilde{D}-r \tilde{L})] x .
$$

Obviously,

$$
\lambda(\widetilde{D}-r \widetilde{L}) x=\lambda(1-r) \widetilde{D} x+\lambda r(\widetilde{D}-\widetilde{L}) x
$$

Hence

$$
\begin{aligned}
\tilde{L}_{r w} x-\lambda x= & (\widetilde{D}-r \widetilde{L})^{-1}[(1-w) \widetilde{D}+(w-r) \widetilde{L}+w \widetilde{U}-\lambda(1-r) \widetilde{D} \\
& -\lambda r(\widetilde{D}-\widetilde{L})] x .
\end{aligned}
$$

Using (E1), by simple manipulations, we get

$$
\begin{aligned}
\tilde{L}_{r w} x-\lambda x= & (\widetilde{D}-r \widetilde{L})^{-1}[(1-\lambda)(1-r) \tilde{D}-(w-r+\lambda r)(\widetilde{D}-\tilde{L}) \\
& +w(\bar{S} U-\bar{S}+U)] x .
\end{aligned}
$$

By (E2) and (E3), the above equation can be written as

$$
\begin{aligned}
\tilde{L}_{r w} x-\lambda x= & (\widetilde{D}-r \widetilde{L})^{-1}[(1-\lambda)(1-r) \widetilde{D}-(w-r+\lambda r)(I-L-\bar{S} L) \\
& -(w-r+\lambda r)(S-S U)+w(I+\bar{S}) U-w \bar{S}] x \\
= & (\widetilde{D}-r \widetilde{L})^{-1}\{(1-\lambda)(1-r) \widetilde{D}-(w-r+\lambda r)(I-L-\bar{S} L) \\
& +(I+\bar{S})[(\lambda-1+w) I-(w-r+\lambda r) L]-(w-r+\lambda r) S \\
& +(w-r+\lambda r) S U-w \bar{S}\} x \\
= & (\widetilde{D}-r \widetilde{L})^{-1}[(1-\lambda)(1-r)(\widetilde{D}-I)-(1-\lambda) \bar{S}-(w-r+\lambda r) S \\
& +(w-r+\lambda r) S U] x \\
= & (\widetilde{D}-r \widetilde{L})^{-1}\{(1-\lambda)(1-r)(\widetilde{D}-I)-(1-\lambda) \bar{S}-(w-r+\lambda r) S \\
& -r(1-\lambda) S U+S[(\lambda+w-1) I-(w-r+\lambda r) L]\} x \\
= & (\widetilde{D}-r \widetilde{L})^{-1}[(1-\lambda)(1-r)(\widetilde{D}-I)-(1-\lambda) \bar{S}-(1-\lambda)(1-r) S \\
& -r(1-\lambda) S U] x .
\end{aligned}
$$

We are now ready to deduce (1)-(3). 
(1) If $0<\lambda<1$; then $\widetilde{L}_{r w} x-\lambda x \leq 0$ but is not equal to the null vector. Therefore $\widetilde{L}_{r w} x \leq \lambda x$. By Lemma 2.5 , we get $\rho\left(\tilde{L}_{r w}\right)<\lambda=\rho\left(L_{r w}\right)$.

(2) If $\lambda=1$, then $\widetilde{L}_{r w} x-\lambda x=0$. By Lemma 2.5 , we get $\rho\left(\tilde{L}_{r w}\right)=\lambda=\rho\left(L_{r w}\right)$.

(3) If $\lambda>1$, then $\widetilde{L}_{r w} x-\lambda x \geq 0$ but is not equal to the null vector. Therefore $\widetilde{L}_{r w} x \geq \lambda x$. By Lemma 2.5 , we get $\rho\left(\widetilde{L}_{r w}\right)>\lambda=\rho\left(L_{r w}\right)$.

Here, we can also construct the preconditioner. That is,

$$
\widehat{S}=\left[\begin{array}{ccccc}
0 & -l_{1} a_{12} & 0 & \cdots & 0 \\
0 & 0 & -l_{2} a_{23} & \cdots & 0 \\
\vdots & \vdots & \vdots & \cdots & \vdots \\
0 & 0 & 0 & \cdots & -l_{n-1} a_{n-1, n} \\
-l_{n} a_{n 1} & 0 & 0 & \cdots & 0
\end{array}\right],
$$

for the preconditioned linear system,

$$
\widehat{A} x=\widehat{b} .
$$

The corresponding preconditioned AOR iterative method has iterative matrices

$$
\widehat{L}_{r w}=(\widehat{D}-r \widehat{L})^{-1}[(1-w) \widehat{D}+(w-r) \widehat{L}+w \widehat{U}] .
$$

Analogously, we have the following lemma and theorem.

LEMMA 4.3. Let $A$ and $\widehat{A}$ be the coefficient matrices of the linear systems (1.1) and (4.2), respectively. If $0 \leq r \leq w \leq 1(w \neq 0, r \neq 1), A$ is an irreducible L-matrix with $0<l_{n} a_{1 n} a_{n 1}<1$ and there exists a nonempty set of $\beta \subseteq N=\{1,2, \ldots, n-1\}$ such that

$$
\begin{cases}0<l_{i} a_{i, i+1} a_{i+1, i}<1, & i \in \beta, \\ a_{i, i+1} a_{i+1, i}=0, & i \in N \backslash \beta .\end{cases}
$$

Then the iterative matrices $L_{r w}$ and $\widehat{L}_{r w}$ associated to the AOR method applied to the linear systems (1.1) and (4.2), respectively, are nonnegative and irreducible.

THEOREM 4.4. Let $L_{r w}$ and $\widehat{L}_{r w}$ be the iterative matrices of the AOR method given by (1.2) and (4.3), respectively. If $0 \leq r \leq w \leq 1(w \neq 0, r \neq 1), A$ is an irreducible $L$-matrix with $0<l_{n} a_{1 n} a_{n 1}<1$ and there exists a nonempty set of $\beta \subseteq N=\{1,2, \cdots, n-1\}$ such that .

$$
\begin{cases}0<l_{i} a_{i, i+1} a_{i+1, i}<1, & i \in \beta, \\ a_{i, i+1} a_{i+1, i}=0, & i \in N \backslash \beta .\end{cases}
$$

Then 
(1) $\rho\left(\widehat{L}_{r w}\right)<\rho\left(L_{r w}\right)$, if $\rho\left(L_{r w}\right)<1$;

(2) $\rho\left(\widehat{L}_{r w}\right)=\rho\left(L_{r w}\right)$, if $\rho\left(L_{r w}\right)=1$;

(3) $\rho\left(\widehat{L}_{r w}\right)>\rho\left(L_{r w}\right)$, if $\rho\left(L_{r w}\right)>1$.

The proof of Theorem 4.4 is similar to that of Theorem 4.2, and is omitted here.

THEOREM 4.5. Let $0<r_{1}<r_{2} \leq w \leq 1$ and $A^{-1} \geq 0$. Under the hypothesis of Theorem 4.2, then $0<\rho\left(\widetilde{L}_{w, r_{2}}\right)<\rho\left(\tilde{L}_{w, r_{1}}\right)<1$, if $0<\bar{\lambda}<1$.

PROOF. Let

$$
\tilde{A}=\tilde{M}_{w, r}-\tilde{N}_{w, r}
$$

where $\tilde{M}_{w, r}=(1 / w)(\tilde{D}-r \tilde{L})$ and $\tilde{N}_{w, r}=(1 / w)[(1-w) \tilde{D}+(w-r) \tilde{L}+w \tilde{U}]$. Since $0<r_{1}<r_{2} \leq w \leq 1$, then $0 \leq \widetilde{N}_{w, r_{2}} \leq \widetilde{N}_{w, r_{1}}$. In terms of Lemma 2.6, this completes the proof.

Subsequently, we have the following theorem.

THEOREM 4.6. Let $0<r_{1}<r_{2} \leq w \leq 1$ and $A^{-1} \geq 0$. Under the hypothesis of Theorem 4.4, then $0<\rho\left(\widehat{L}_{w, r_{2}}\right)<\rho\left(\widehat{L}_{w, r_{1}}\right)<1$, if $0<\bar{\lambda}<1$.

REMARK 2. From the previous results, it is easy to reach the conclusion that convergence of the preconditioned SOR iterative method is faster than that of the preconditioned AUR iterative method. That is, from the preconditioned AOR, we find that the optimal value of $r$ is equal to $w$.

It is well known that, when $w=r$, AOR iteration reduces to SOR iteration. So we can easily get the following corollaries.

COROLLARY 4.7. Let $L_{w}$ and $\widetilde{L}_{w}$ be the iterative matrices of the SOR iterative method associated to (1.1) and (3.1), respectively. If $0<w<1, A$ is an irreducible $L$ matrix with $0<a_{1 n} a_{n 1}<1$ and there exists a nonempty set of $\alpha \subseteq N=\{1,2, \ldots, n-$ 1) such that

$$
\begin{cases}0<a_{i, i+1} a_{i+1, i}<1, & i \in \alpha, \\ a_{i, i+1} a_{i+1, i}=0, & i \in N \backslash \alpha .\end{cases}
$$

Then

(1) $\rho\left(\tilde{L}_{w}\right)<\rho\left(L_{w}\right)$ if $\rho\left(L_{w}\right)<1$;

(2) $\rho\left(\widetilde{L}_{w}\right)=1$ if $\rho\left(L_{w}\right)=1$;

(3) $\rho\left(\tilde{L}_{w}\right)>\rho\left(L_{w}\right)$ if $\rho\left(L_{w}\right)>1$. 
COROLLARY 4.8. Let $L_{w}$ and $\widehat{L}_{w}$ be the iterative matrices of the SOR iterative method associated to (1.1) and (4.2), respectively. If $0<w<1, A$ is an irreducible $L$-matrix with $0<l_{n} a_{1 n} a_{n 1}<1$ and there exists a nonempty set of $\beta \subseteq N=$ $\{1,2, \cdots, n-1\}$ such that

$$
\begin{cases}0<l_{i} a_{i, i+1} a_{i+1, i}<1, & i \in \beta, \\ a_{i, i+1} a_{i+1, i}=0, & i \in N \backslash \beta .\end{cases}
$$

Then

(1) $\rho\left(\widehat{L}_{w}\right)<\rho\left(L_{w}\right)$ if $\rho\left(L_{w}\right)<1$;

(2) $\rho\left(\widehat{L}_{w}\right)=1$ if $\rho\left(L_{w}\right)=1$;

(3) $\rho\left(\widehat{L}_{w}\right)>\rho\left(L_{w}\right)$ if $\rho\left(L_{w}\right)>1$.

COROLLARY 4.9. Let $0<w_{1}<w_{2} \leq 1$ and $A^{-1} \geq 0$. Under the hypothesis of Corollary 4.7, then $0<\rho\left(\widetilde{L}_{w_{2}}\right)<\rho\left(\widetilde{L}_{w_{1}}\right)<1$, if $0<\lambda<1$.

COROLlaRY 4.10. Let $0<w_{1}<w_{2} \leq 1$ and $A^{-1} \geq 0$. Under the hypothesis of Corollary 4.8 , then $0<\rho\left(\widehat{L}_{w_{2}}\right)<\rho\left(\widehat{L}_{w_{1}}\right)<1$, if $0<\lambda<1$.

REMARK 3. From the above results, it is easy to reach the conclusion that convergence of the preconditioned Gauss-Seidel iterative method is faster than that of the preconditioned SOR iterative method, that is, $w=1$ is the optimal value.

\section{Numerical example}

Now let us consider the following example to illustrate the results. Suppose that the coefficient matrix $A$ of $(1.1)$ is given by

$$
A=\left[\begin{array}{cccccc}
1 & q & r & s & q & \cdots \\
s & 1 & q & r & \ddots & q \\
q & s & 1 & q & \ddots & s \\
r & q & s & 1 & \ddots & r \\
s & \ddots & \ddots & \ddots & \ddots & q \\
\cdots & s & r & q & s & 1
\end{array}\right],
$$

where $q=-p / n, r=-p /(n+1)$ and $q=-p /(n+2)$. For $n=6$ and $p=1$, Table 1 displays the spectral radius of the corresponding iterative matrix with different parameters $w$ and $r$. These calculations were performed using Matlab 7.1. For convenience, we denote by $\ell_{w r}$ the iterative matrix under the conditions of Theorem 2.2 in [2] and by $\bar{L}_{w r}$ the iterative matrix under the conditions of Theorem 2 in [6]. 
TABLE 1. Numerical illustration of Theorem 4.2.

\begin{tabular}{|l|l|l|c|c|c|}
\hline$w$ & $r$ & $\rho\left(\widetilde{L}_{w r}\right)$ & $\rho\left(\bar{L}_{w r}\right)$ & $\rho\left(\ell_{w r}\right)$ & $\rho\left(L_{w r}\right)$ \\
\hline 0.9 & 0.8 & 0.5606 & 0.5768 & 0.6367 & 0.6519 \\
\hline 0.95 & 0.8 & 0.5362 & 0.5533 & 0.6165 & 0.6325 \\
\hline 0.8 & 0.7 & 0.6365 & 0.6492 & 0.6962 & 0.7083 \\
\hline 0.7 & 0.65 & 0.6921 & 0.7026 & 0.7416 & 0.7517 \\
\hline
\end{tabular}

REMARK 4. Table 1 also illustrates that convergence of the AOR iterative method is faster than that of the AOR iterative method of [2] and [6].

REMARK 5. When $n=6$ and $p=1$, we get Tables 3 and 4 by our numerical experiments.

TABLE 2. Numerical illustration of Theorem 4.4.

\begin{tabular}{|c|l|l|l|l|}
\hline$l$ & $w$ & $r$ & $\rho\left(\widehat{L}_{w r}\right)$ & $\rho\left(L_{w r}\right)$ \\
\hline$(1,1,2,1,1,1)$ & 0.7 & 0.65 & 0.6975 & 0.7517 \\
\hline$(2,1,3,1,1,2)$ & 0.95 & 0.85 & 0.5476 & 0.6205 \\
\hline$(1,4,1,3,5,2)$ & 0.9 & 0.86 & 0.5844 & 0.6381 \\
\hline$(2,4,5,3,6,8)$ & 0.8 & 0.75 & 0.6809 & 0.6998 \\
\hline
\end{tabular}

TABLE 3. Numerical illustration of Theorem 4.5.

\begin{tabular}{|l|l|l|l|}
\hline$w$ & $r$ & $\rho\left(\widetilde{L}_{w r}\right)$ & $\rho\left(L_{w r}\right)$ \\
\hline 0.9 & 0.5 & 0.7748 & 0.8014 \\
\hline 0.9 & 0.6 & 0.7602 & 0.7899 \\
\hline 0.9 & 0.8 & 0.7227 & 0.7615 \\
\hline 0.9 & 0.9 & 0.6977 & 0.7433 \\
\hline
\end{tabular}

\section{Conclusion}

In this paper, we have presented an accelerating algorithm for iteratively solving linear systems based on the AOR (SOR) methods. It remains to construct a comparison theorem for accurate estimation of the optimum parameter. Meanwhile, we obtain that convergence of the modified Gauss-Seidel method is faster than that of the modified AOR (SOR) iterative method for $L$-matrix linear systems. 
TABLE 4. Numerical illustration of Corollary 4.9.

\begin{tabular}{|l|l|l|l|}
\hline$w$ & $r$ & $\rho\left(\widetilde{L}_{w r}\right)$ & $\rho\left(L_{w r}\right)$ \\
\hline 0.1 & 0.1 & 0.9798 & 0.9818 \\
\hline 0.3 & 0.3 & 0.9329 & 0.9402 \\
\hline 0.8 & 0.8 & 0.7535 & 0.7880 \\
\hline 1 & 1 & 0.6284 & 0.6901 \\
\hline
\end{tabular}

\section{Acknowledgements}

The authors would like to thank the referee and Associate Editor Dr B. D. Craven very much for his extremely helpful suggestions for improving the presentation of this manuscript.

This research was supported by NSFC under Grant (10771030), the Scientific and Technological Key Project of the Chinese Ministry of Education under Grant (107098), the PhD Programs Fund of Chinese Universities under Grant (20070614001) and the Project for Academic Leader and Group of UESTC.

\section{References}

[1] A. Berman and R. J. Plemmons, Nonnegative matrices in the mathematical sciences (SIAM, Philadelphia, PA, 1994).

[2] D. J. Evans, M. M. Martins and M. E. Trigo, "The AOR iterative method for new preconditioned linear systems", J. Comput. Appl. Math. 132 (2001) 461-466.

[3] A. D. Gunawardena, S. K. Jain and L. Snyder, "Modified iteration methods for consistent linear systems", Linear Algerbra Appl. 154-156 (1991) 123-143.

[4] A. Hadjimos, "Accelerated overelaxation method", Math. Comp. 32 (1978) 149-157.

[5] T. Z. Huang, G. H. Cheng and X. Y. Cheng, "Modified SOR-type iterative method for Z-matrices", Appl. Math. Comput. 175 (2006) 258-268.

[6] Y. T. Li, C. X. Li and S. L. Wu, "Improving AOR method for consistent linear systems", Appl. Math. Comput. 186 (2007) 379-388.

[7] J. P. Milaszewicz, "Improving Jacobi and Gauss-Seidel iterations", Linear Algebra Appl. 93 (1987) 161-170.

[8] R. S. Varga, Matrix iterative analysis (Prentice-Hall, Englewood Cliffs, New York, 1962).

[9] Z. D. Wang and T. Z. Huang, "The upper Jacobi and upper Gauss-Seidel type iterative methods for preconditoned linear systems", Appl. Math. Lett. 19 (2006) 1029-1036.

[10] D. M. Young, Iterative solution of large linear systems (Academic Press, New York-London, 1971). 